\title{
Applications of Ultrafast Terahertz Pulses for Intra-Excitonic Spectroscopy of Quasi-2D Electron-Hole Gases
}

\author{
Robert A. Kaindl , Marc A. Carnahan, Daniel Hägele ${ }^{\dagger}$, and D. S. Chemla \\ Department of Physics, University of California at Berkeley and Materials Sciences Division, \\ E. O. Lawrence Berkeley National Laboratory, \\ 1 Cyclotron Road, Berkeley, CA 94720, USA \\ Phone: +1 (510) 486 7475, Fax: +1 (510) 486 6695, E-mail: RAKaindl@lbl.gov
}

\begin{abstract}
Excitons are of fundamental interest and of importance for opto-electronic applications of bulk and nano-structured semiconductors. This paper discusses the utilization of ultrafast terahertz $(\mathrm{THz})$ pulses for the study of characteristic low-energy excitations of photoexcited quasi-2D electron-hole $(e-h)$ gases. Optical-pump THz-probe spectroscopy at 250-kHz repetition rate is employed to detect characteristic $\mathrm{THz}$ signatures of excitons and unbound $e-h$ pairs in GaAs quantum wells. Exciton and free-carrier densities are extracted from the data using a two-component model. We report the detailed $\mathrm{THz}$ response and pair densities for different photoexcitation energies resonant to heavy-hole excitons, light-hole excitons, or the continuum of unbound pairs. Such experiments can provide quantitative insights into wavelength, time, and temperature dependence of the low-energy response and composition of optically excited $e$ - $h$ gases in low-dimensional semiconductors.
\end{abstract}

Keywords: Low-dimensional Semiconductors, Terahertz Spectroscopy, Excitons, Ultrafast Processes

\footnotetext{
* Author to whom correspondence should be addressed.

${ }^{\dagger}$ Present address: Institut für Festkörperphysik , Universität Hannover, Appelstraße 2, 30167 Hannover, Germany
} 


\section{INTRODUCTION}

Understanding the collective physical properties of complex many-particle systems is one of the most important problems of condensed matter physics. Coulomb interactions between a large number of particles can result in the appearance of novel quantum phases, such as exciton gases, metal-insulator transitions, fractionally charged states, or Bose-Einstein and superconducting condensates. Excitons, in particular, are intrinsically non-equilibrium in nature and are important for opto-electronic applications. The same forces that form such many-body states also govern their interactions on femtosecond and picosecond timescales. Significant insight into microscopic processes and non-equilibrium states can be obtained from ultrafast time-resolved spectroscopy ${ }^{1,2}$.

A focus of recent experiments, including our own, has been the detection of transient changes in the terahertz (THz) response of semiconductors and superconductors ${ }^{3-10}$. As $1 \mathrm{THz}$ corresponds to $\approx 4.1 \mathrm{meV}$ photon energy, the $\mathrm{THz}$ spectral region matches the energy scale of thermal excitations. It thus provides access to low-energy excitations that are directly relevant to the basic physics of nano-scale and bulk materials. In this paper, we discuss THz studies of excitons and free-carriers in photoexcited quasi-2D electron-hole $(e-h)$ gases.

In the past, excitons have been extensively studied using optical absorption and photoluminescence near the semiconductor bandgap ${ }^{11}$. However, these techniques are limited by the vanishing momentum of the near-infrared photons. This typically restricts their sensitivity to a subset of excitons around center-of-mass momentum $K \approx 0$ (see Fig. 1). Extracting absolute exciton densities from luminescence is thus difficult, even for thermal exciton populations where signals are influenced by both the density and temperature of the 
exciton gas. The problem is exacerbated in the case of nonthermal populations or for optically“dark” excitons, candidate systems for the long-sought excitonic Bose-Einstein condensates, which neccessarily couple only weakly to interband resonances.

Terahertz transitions between internal levels of excitons constitute a fundamentally different tool to measure these quasi-particles. Such intra-excitonic transitions have been only

scarcely investigated in the past. ${ }^{12-15}$ Recent advances in coherent $\mathrm{THz}$ spectroscopy provide new opportunities to study the densities, internal structure, and dynamics of excitons ${ }^{7,} 16$. Intra-excitonic transitions are not restricted by the exciton's center of mass momentum, because they couple only states with different relative momentum of the $e$-h pairs (Fig. 1). Therefore, they are a sensitive gauge of absolute pair densities. This underscores the importance of intra-excitonic THz spectroscopy as a powerful tool for exciton spectroscopy.

In the following, we discuss applications of ultrafast $\mathrm{THz}$ probes for the study of quasi2D $e$-h gases in GaAs quantum wells. Important spectral differences exist in the low-energy response of excitons and free carriers, which enables separation of their relative contributions. We provide examples where the transient changes of the $\mathrm{THz}$ conductivity provide insight into the composition of the photoexcited $e-h$ gases as a function of time, temperature, and pump photon energy.

\section{OPTICAL-PUMP TERAHERTZ-PROBE SPECTROSCOPY}

The setup used for our experiments is illustrated in Fig. 2. At the outset, a commercially available high-repetition rate regenerative Ti:sapphire amplifier (Coherent RegA 9000) produces 150 -fs pulses centered around $800 \mathrm{~nm}$ wavelength. For many optical-pump $\mathrm{THz}$ probe experiments, a pump energy in the 10-100 nJ range is necessary to fill out the 
intrinsically large ( $\mathrm{mm}^{2}$-sized) focal spots of the long-wavelength $\mathrm{THz}$ beam. The $250-\mathrm{kHz}$ repetition rate then provides for an optimal balance between high probe sensitivity and sufficient pump pulse energy.

About $20 \%$ of the amplifier output is used for $\mathrm{THz}$ generation and detection. Specifically, optical rectification in a $500-\mu \mathrm{m}$ thick $<110>$ oriented ZnTe crystal results in broadband, subpicosecond $\mathrm{THz}$ pulses that span the 2-12 meV spectral range. Next, the THz beam is collimated and refocused using off-axis parabolic mirrors, while silicon filters block out any residual near-infrared light. The THz electric field is then detected coherently in the time-domain via electro-optic sampling in a second 500- $\mu$ m thick $<110>$ ZnTe crystal. In this scheme ${ }^{17}$ a linearly-polarized "sampling pulse” of 150 fs duration travels collinearly with the THz pulse. During propagation in the thin ZnTe crystal, the sampling pulse encounters only a small temporal slice of the THz field. For each THz field delay $t$ (Fig. 2), the Pockels nonlinearity in $\mathrm{ZnTe}$ results in a polarization rotation that is proportional to the momentary THz electric field $E(t)$. By measuring the polarization rotation angle as a function of the delay $t$, the THz field $E(t)$ is mapped out directly in the time domain.

For the pump-probe experiments, a time-delayed second portion of the amplifier output photoexcites the sample. To cover specific resonances, the wavelength and spectral width of the pump pulses can be tailored using a grating-based pulse shaper. The transmitted pump light is picked up by a spectrometer and diode array to enable optimization of the photoexcitation conditions. After photoexcitation of the sample, its equilibrium THz dielectric function $\varepsilon(\omega)$ changes by an incremental amount $\Delta \varepsilon(\omega)$. For each fixed time delay $\Delta t$ between the optical pump and THz probe pulses, we measure the pump-induced change $\Delta E(t)$ in the transmitted THz reference field. Fourier transformation results in complex-valued fields in frequency 
space. We then numerically obtain the induced change $\Delta \varepsilon(\omega)$ from $E(\omega)$ and $\Delta E(\omega)$ after taking into account the sample's layer sequence and resulting transmission function via the

well-known transfer matrix approach ${ }^{18,19}$. Time-domain measurement of the THz fields yields real and imaginary parts of the response directly, without the need for Kramers-Kronig transforms.

In the following, we will express the $\mathrm{THz}$ response through the optical conductivity $\sigma(\omega)$ which describes the high-frequency current response of the many-particle system. It will be useful to write the complex-valued conductivity as $\sigma(\omega)=\sigma_{1}(\omega)+i \omega \varepsilon_{0}\left[1-\varepsilon_{1}(\omega)\right]$. The real part of conductivity, $\sigma_{1}$, is a measure of the absorbed power density. The imaginary conductivity is expressed through the real part of the dielectric function, $\varepsilon_{1}$, which in turn describes the out-of-phase, inductive response. As discussed below, the availability of both $\sigma_{1}$ and $\varepsilon_{1}$ is important to distinguish different components of the photoexcited response.

\section{INTRA-EXCITONIC TRANSITIONS: EXPERIMENT AND THEORY}

In the following, we discuss the transient low-energy response of photoexcited quasi-2D $e-h$ gases. As reported earlier, a particularly important application for the ultrafast $\mathrm{THz}$ probe is the study of exciton formation and ionization dynamics ${ }^{7}$. Our sample is a high-quality MBEgrown multiple-quantum well structure, which consists of ten 14-nm wide GaAs quantum wells separated by $10-\mathrm{nm}$ thick AlGaAs barriers. The GaAs substrate was removed through etching to prevent unwanted excitation of free carriers in the bulk. The near-infrared absorption spectrum at $T=6 \mathrm{~K}$ is shown on the right hand side in Fig. 1: it consists of a 0.8-meV wide $1 s$ heavy-hole $(\mathrm{HH})$ line centered at $1.54 \mathrm{eV}$, and of $2 s-\mathrm{HH}$ and 1 s light-hole (LH) exciton peaks at higher energies, followed by the broadband interband continuum. For 
selective excitation of different excitons or the continuum in the experiments, we tailor the tunable pump pulses to $\approx 2 \mathrm{meV}$ bandwidth. Figure 3 displays transient changes of the $\mathrm{THz}$ response which are observed after resonant excitation of 1s-HH excitons. A strong peak occurs around $7 \mathrm{meV}$ photon energy (1.7 THz) in the induced THz conductivity $\Delta \sigma_{1}$, followed by a shoulder at higher photon energies. This corresponds to the appearance of a new low-energy oscillator, which is confirmed by the dispersive dielectric function change $\Delta \varepsilon_{1}$ at the same energy. This $\mathrm{THz}$ response arises from intra-excitonic transitions, dominated by the $1 s \rightarrow 2 p$ transition at $7 \mathrm{meV}$. The exciton response in Fig. 3 exhibits a vanishing low-frequency conductivity which confirms the charge-neutral, insulating nature of pure exciton gases.

We have performed model calculations of the intra-excitonic $\mathrm{THz}$ response. The contribution to the $\mathrm{THz}$ dielectric function for a $1 s$ exciton population is expressed as

$$
\Delta \varepsilon(\omega)=n_{X} \Delta \varepsilon_{X}(\omega)=n_{X} \cdot \frac{2 e^{2}}{\hbar \varepsilon_{0}} \sum_{f} \frac{\omega_{1 s \rightarrow f}}{\omega_{1 s \rightarrow f}^{2}-\omega^{2}-i \omega \Gamma}\left|\left\langle\psi_{f}|x| \psi_{1 s}\right\rangle\right|^{2},
$$

where $n_{\mathrm{X}}$ is the exciton density and $\Delta \varepsilon_{\mathrm{X}}(\omega)$ is the response of a single exciton. The expression includes a sum over the transitions to all bound and continuum final states $f$, with related transition energies $\omega_{1 \mathrm{~s} \rightarrow f}$ and broadening $\Gamma$. Two broadening parameters are used: one for all bound-bound transitions (adapted to the experimentally observed width), and a boundcontinuum broadening of $2 \mathrm{meV}$ in accordance with the typical Drude widths observed after nonresonant excitation. The matrix element is calculated for electric field polarization parallel to the coordinate $x$ in the quantum well plane. For the wavefunction $\psi_{1 s}$ of the $1 s$ ground state and for the bound and continuum final states $\psi_{f}$, we take into account the non-integer (quasi-2D) dimensionality of the Coulomb interaction following the work of Ekenberg ${ }^{20}$. As evident from Fig. 3, the calculated response (solid lines) is in excellent agreement with the 
experimental observations. A reduced mass $\mu=0.054 m_{0}$ and dielectric constant $\varepsilon_{\mathrm{s}}=13.2$ was used in agreement with known values for this material system. The model confirms that the peak arises from the $1 s-2 p$ transition, while the shoulder stems from transitions into higher bound and continuum states.

An important advantage of THz spectroscopy of $e$ - $h$ gases is the absolute scaling of the response with carrier density. Within a parabolic band approximation, we can write a partial oscillator strength sum rule

$$
S \equiv \int_{0}^{E_{C}} \sigma_{1}(\omega) d \omega=\frac{\pi}{2} \frac{n_{e} e^{2}}{m_{e}^{*}}+\frac{\pi}{2} \frac{n_{h} e^{2}}{m_{h}^{*}}=\frac{\pi}{2} \frac{n e^{2}}{\mu} .
$$

Here, $n_{\mathrm{e}}$ and $n_{\mathrm{h}}$ are the three-dimensional electron and hole densities, respectively, and $m_{\mathrm{e}}{ }^{*}$ and $m_{\mathrm{h}}{ }^{*}$ the corresponding effective masses. The last equality above holds for equal densities of electrons and holes, such that the pair density can be written as $n \equiv n_{\mathrm{e}}=n_{\mathrm{h}}$, and the reduced mass is defined as usual as $\mu \equiv m_{\mathrm{e}}{ }^{*} m_{\mathrm{h}}{ }^{*} /\left(m_{\mathrm{e}}{ }^{*}+m_{\mathrm{h}}{ }^{*}\right)$. This partial sum rule states that the intraband spectral weight - given by the integral of $\sigma_{1}(\omega)$ to cut-off energies $E_{C}$ just below the interband transitions - is determined only by the pair density $n$ and reduced mass $\mu$ of the $e-h$ pairs. Conversely, the pair density can be inferred directly from the $\mathrm{THz}$ measurements for most materials where the reduced mass is well known. This stands in stark contrast to the difficulties of photoluminescence measurements mentioned above. We note that the intraexcitonic model discussed above precisely fulfills Eq. (2), which makes it a powerful tool to gauge absolute exciton densities. 


\section{NON-RESONANT PHOTOEXCITATION}

Photoexcitation into the continuum of unbound states leads to a broadband $\mathrm{THz}$ response which strongly contrasts with the structured intra-excitonic spectrum described above. The room-temperature response ( $T=300 \mathrm{~K})$ is shown in Fig. 4(a). It exhibits a large low-frequency conductivity $\Delta \sigma_{1}$, signifying a conducting state, accompanied by a strongly dispersive dielectric function change $\Delta \varepsilon_{1}$ that approximately follows a $-1 / \omega^{2}$ functional shape. This response is well explained by the Drude model

$$
\Delta \varepsilon(\omega)=n_{e h} \Delta \varepsilon_{e h}(\omega)=n_{e h}\left(\frac{e^{2}}{\mu \varepsilon_{0}} \cdot \frac{-1}{\omega^{2}+i \omega \Gamma_{D}}\right)
$$

where $n_{\mathrm{eh}}$ is the free carrier density, and $\Gamma_{\mathrm{D}}$ is the Drude scattering rate. Note that both $n_{\mathrm{eh}}$ and $n_{\mathrm{X}}$ are three-dimensional quantities in the equations and are converted into 2D sheet densities after division through the quantum well width. A Drude model curve is shown in Fig. 4(a) as solid lines, and corroborates the good agreement with the experimental data. As is evident directly from the data in Fig. 4(a), the photoinduced Drude response persists on a ns timescale with only minor variation in the studied range up to 1000 ps.

Significant changes occur in the $\mathrm{THz}$ response of the non-resonantly excited quantum wells, however, when the lattice temperature is reduced. Figure 4(b) shows the transient spectra at $T=6 \mathrm{~K}$. Directly after excitation ( $\Delta t=0 \mathrm{ps}$ ), a broadband $\mathrm{THz}$ response is observed. The large low-frequency conductivity $\Delta \sigma_{1}$ and dispersive, negative dielectric function change $\Delta \varepsilon_{1}$ identify a significant Drude-like component. This presence of a conducting gas of unbound $e-h$ pairs is not unexpected given the nonresonant excitation. However, the $\mathrm{THz}$ conductivity $\Delta \sigma_{1}$ also strongly deviates at higher frequencies from the simple Drude model shown as the 
dotted lines. This reveals a strong excitonic peak which appears quasi-instantaneously within our time-resolution after nonresonant excitation.

The subsequent time evolution in Fig.4(b) on a comparatively long timescale of several 100 ps shows the continued growth of the intra-excitonic peak, accompanied by a loss of the free-carrier Drude component. Moreover, the initally large broadening of the intraexcitonic peak decreases with time. At $\Delta t=1000 \mathrm{ps}$, the characteristic response of a pure $1 \mathrm{~s}$ exciton population is restored, with only a minimal Drude component surviving. Figure 4(b) thus directly evidences the process of exciton formation. A particularly interesting aspect is the manifestation of two distinct timescales: (i) the quasi-instantaneous formation of a significant fraction of excitons, within the $\approx 1$ ps time-resolution of our experiment, and (ii) a slower transformation of the overall mixture of bound and unbound $e$-h pairs into a pure exciton gas. Since the femtosecond near-infrared pump pulse exclusively photoexcites unbound pairs, the sub-ps apperance of the intra-excitonic peak points to a very efficient exciton formation mechanism. In particular, ultrafast Coulomb interactions between $e$-h pairs and with optical phonons can contribute to this effect. In contrast, the slow scattering of free carriers into excitons on a 100 ps timescale can be explained by emission of acoustic phonons ${ }^{21,22}$. The full transformation into a pure exciton gas necessitates the irreversible energy transfer into the phonon modes.

We can obtain a quantitative description of the mixed state observed during exciton formation through a two-component model of the dielectric response:

$$
\Delta \varepsilon(\omega)=n_{e h} \Delta \varepsilon_{e h}(\omega)+n_{X} \Delta \varepsilon_{X}(\omega) .
$$

Model curves are shown as solid black lines in Fig. 4(b). They reproduce all the main features of the data and provide a direct absolute scaling of the exciton and free carrier densities. The 
excitonic component is also separately shown as dashed lines in Fig. 4(b). Moreover, with the knowledge of $n_{\text {eh }}$ and $n_{\mathrm{X}}$, we immediately obtain the exciton fraction $f \equiv n_{\mathrm{X}} /\left(n_{\mathrm{eh}}+n_{\mathrm{X}}\right)$ of the mixed state. Directly after excitation, it is $f \approx 42 \%$, which underscores the fast initial conversion of free carriers into excitons. The exciton fraction then increases with time from $f \approx 52 \%$ (20 ps), via $75 \%$ (200 ps) and $84 \%$ (700 ps), up to $90 \%$ at 1000 ps.

\section{PUMP PHOTON DEPENDENCE}

In the following, we report the detailed dependence of the $\mathrm{THz}$ response on the pump photon energy $E_{\text {pump }}$ in the near-bandgap region. Figure 5 shows the induced changes for different $\mathrm{E}_{\text {pump }}$ and for three pump-probe delays each, as indicated.

Resonant excitation at different positions within the $1 \mathrm{~s} \mathrm{HH}$ exciton line is shown in the top three sets of curves in Fig. 5. The exciton population significantly decays between 0 ps (magenta) and $200 \mathrm{ps}$ (green) after excitation at the low-energy wing of the $1 \mathrm{~s} \mathrm{HH}$ peak ( $\mathrm{E}_{\text {pump }}$ $=1.5398 \mathrm{eV}$ and $1.5412 \mathrm{eV})$. In contrast, for excitation at the high-energy wing $(1.5421 \mathrm{eV})$ this fast decay is absent. The intra-excitonic line also clearly broadens with increasing pump photon energy. The differences can be understood by the generation of a hot exciton distribution when excitation occurs in the high energy region of the $1 \mathrm{~s} \mathrm{HH}$ line. The excitons then occupy regions outside the optical cone, which decreases their recombinative population decay.

For photoexcitation at the $2 \mathrm{~s} \mathrm{HH}$ exciton line at $1.5477 \mathrm{eV}$, the response already directly after excitation ( $0 \mathrm{ps}$, magenta curve) corresponds to a conducting state with a significant Drude-like dispersion in $\Delta \varepsilon_{1}$. This clearly evidences a fast and efficient dissociation of the higher-bound excitons, which can occur via phonon absorption since the small binding 
energy of the $2 s$ state is comparable to the $T=6 \mathrm{~K}$ thermal excitation energy. However, a significant exciton fraction does coexist at 0 ps. Exciton re-formation then takes place with increasing time delay, such that finally at $900 \mathrm{ps}$ the $e$ - $h$ gas consists predominantly of excitons (blue curve, $1.5477 \mathrm{eV})$. Excitation at the LH peak (1.5521 eV) and in the continuum (1.5590 eV) also shows the characteristic dynamics of exciton formation discussed previously.

Curves from the two-component model were generated for each experimentally measured dataset (and for additional time delays not shown) and are indicated by the solid lines in Fig. 5. The corresponding pair densities and exciton fractions obtained in this way are reported in Fig. 6. The total pair density is shown in Fig. 6(a) as black squares. It agrees well with the densities expected from the near-infrared absorption spectrum (red line) which has been convoluted with the finite spectral width of the pump pulses. The pump-photon energy dependent $\mathrm{THz}$ spectrum is analogous to photoluminescence excitation spectroscopy, but it provides direct information about the absolute pair densities and composition of the $e-h$ gases.

The free carrier and exciton densities are shown in Figs. 6(b),(c) for two different time delays. Figure 6(b) again evidences the mixed nature of the e-h gas directly after photoexcitation into higher bound and continuum states, while a pure exciton population is obtained initially only for resonant 1 s HH excitation. At long time delays (900 ps, Fig. 6c), almost all free carriers have decayed or formed excitons. The system then consists almost exclusively of excitons. As is also evident from Fig. 6c, the surviving pair density for resonant and nonresonant excitation is now of comparable magnitude, in contrast to the initial photoexcited pair densities. This can be explained by a longer recombination time of free carriers as compared to their exciton counterparts. 
Figure 6(d) provides a comprehensive overview of the pump-photon energy and time dependence of the exciton fraction. The exciton formation process is particularly apparent from this data. A slight enhancement is observed at the $1 \mathrm{~s} \mathrm{LH}$ energy, although for this resonant light-hole excitation a significant free carrier component appears within the time resolution. The latter points to a quick dissociation of light hole excitons, which can be explained by the degeneracy of their energy with the continuum of unbound states.

\section{CONCLUSIONS}

We have discussed experiments that study the low-energy response of photoexcited $e$ - $h$ gases in GaAs quantum wells using ultrashort $\mathrm{THz}$ pulses. In the presence of excitons, intraexcitonic transitions occur with a spectrum that is peaked at the $1 s-2 p$ energy splitting. This characteristic THz fingerprint differs strongly from the conducting Drude response of free carriers. A particular advantage of THz spectroscopy over photoluminescence is that absolute pair densities can be directly obtained. We have illustrated this point by employing a twocomponent model to quantitatively determine exciton and free-carrier densities. In particular, we report in detail the $\mathrm{THz}$ response and time evolution of $e$-h gases while tuning the photoexcitation energy around the bandgap, resonant to $\mathrm{HH}$ excitons, $\mathrm{LH}$ excitons, and the continuum. The analogy to atomic spectroscopy motivates studies which control and probe excitonic level populations ${ }^{23,24}$, enabling for instance stimulated $\mathrm{THz}$ emission from intraexcitonic transitions ${ }^{9}$. The capability of $\mathrm{THz}$ pulses to detect excitons outside the optically accessible range is of increasing interest to the search for excitonic Bose-Einstein

condensates $^{16,25}$. In this way, THz pulses represent a powerful and promising tool to study the ultrafast dynamics and low-energy physics of excitons. 
Acknowledgements: We thank J. Reno for growth of the quantum well samples. This work was supported by the Office of Basic Energy Sciences of the U.S. Department of Energy under Contract No. DE-AC02-05CH11231. R. K. acknowledges support from the Deutsche Forschungsgemeinschaft, D. H. support from the Alexander von Humboldt Foundation. 


\section{References}

1. Ultrafast Phenomena XIV - Proceedings of the 14th International Conference, Springer, Berlin (2005).

2. D. S. Chemla and J. Shah. Many-body and correlation effects in semiconductors. Nature vol. 411, 549 (2001).

3. M. C. Beard, G. M. Turner, and C. A. Schmuttenmaer. Transient photoconductivity in GaAs as measured by time-resolved terahertz spectroscopy. Phys. Rev. B vol. 62, 15764 (2000).

4. R. D. Averitt, G. Rodriguez, A. I. Lobad, J. L. W. Siders, S. A. Trugman, and A. J. Taylor. Nonequilibrium superconductivity and quasiparticle dynamics in $\mathrm{YBa}_{2} \mathrm{Cu}_{3} \mathrm{O}_{7-\delta}$. Phys. Rev. B vol. 63, 140502 (2001).

5. R. A. Kaindl, M. Woerner, T. Elsaesser, D. C. Smith, J. F. Ryan, G. A. Farnan, M. P. McCurry, and D. G. Walmsley. Ultrafast mid-infrared response of $\mathrm{YBa}_{2} \mathrm{Cu}_{3} \mathrm{O}_{7-\delta}$. Science vol. 287, 470 (2000).

6. R. Huber, F. Tauser, A. Brodschelm, M. Bichler, G. Abstreiter, and A. Leitenstorfer. How many-particle interactions develop after ultrafast excitation of an electron-hole plasma. Nature vol. 414, 286 (2001).

7. R. A. Kaindl, M. A. Carnahan, D. Hägele, R. Lövenich, and D. S. Chemla. Ultrafast terahertz probes of transient conducting and insulating phases in an electron-hole gas. Nature vol. 423, 734 (2003).

8. C. W. Luo, K. Reimann, M. Woerner, T. Elsaesser, R. Hey, and K. H. Ploog. PhaseResolved Nonlinear Response of a Two-Dimensional Electron Gas under Femtosecond Intersubband Excitation. Phys. Rev. Lett. vol. 92, 047402 (2004).

9. R. Huber, B. A. Schmid, Y. R. Shen, D. S. Chemla, and R. A. Kaindl. Stimulated Terahertz Emission from Intra-Excitonic Transitions in $\mathrm{Cu}_{2} \mathrm{O}$. Phys. Rev. Lett. vol. 96, 017402 (2006).

10. R. A. Kaindl, M. A. Carnahan, D. S. Chemla, S. Oh, and J. N. Eckstein. Dynamics of Cooper pair formation in $\mathrm{Bi}_{2} \mathrm{Sr}_{2} \mathrm{CaCu}_{2} \mathrm{O}_{8+\delta}$. Phys. Rev. B vol. 72, 060510(R) (2005).

11. J. Shah, Ultrafast Spectroscopy of Semiconductors and Semiconductor Nanostructures, Springer Verlag, Berlin (1999). 
12. R. H. M. Groeneveld and D. Grischkowsky. Picosecond time-resolved far-infrared experiments on carriers and excitons in GaAs-AlGaAs multiple quantum wells. J. Opt. Soc. Am. B vol. 11, 2502 (1994).

13. T. Timusk. Far-infrared absorption study of exciton ionization in germanium. Phys. Rev. B vol. 13, 3511 (1976).

14. J. Cerne, J. Kono, M. S. Sherwin, M. Sundaram, A. C. Gossard, and G. E. W. Bauer. Terahertz Dynamics of Excitons in GaAs/AlGaAs Quantum Wells. Phys. Rev. Lett. vol. 77, 1131 (1996).

15. M. Kira, W. Hoyer, T. Stroucken, and S. W. Koch. Exciton Formation in Semiconductors and the Influence of a Photonic Environment. Phys. Rev. Lett. vol. 87, 176401 (2001).

16. M. Kubouchi, K. Yoshioka, R. Shimano, A. Mysyrowicz, and M. Kuwata-Gonokami. Study of Orthoexciton-to-Paraexciton Conversion in $\mathrm{Cu}_{2} \mathrm{O}$ by Excitonic Lyman Spectroscopy. Phys. Rev. Lett. vol. 94, 016403 (2005).

17. Q. Wu and X.-C. Zhang. Appl. Phys. Lett. vol. 68, 1604 (1996).

18. M. Born and E. Wolf, Principles of Optics, University Press, Cambridge (1999).

19. R. A. Kaindl, D. Hägele, M. A. Carnahan, and D. S. Chemla. (unpublished).

20. U. Ekenberg and M. Altarelli. Exciton binding energy in a quantum well with inclusion of valence-band coupling and nonparabolicity. Phys. Rev. B vol. 35, 7585 (1987).

21. I. K. Oh, J. Singh, A. Thilagam, and A. S. Vengurlekar. Exciton formation assisted by LO phonons in quantum wells. Phys. Rev. B vol. 62, 2045 (2000).

22. K. Siantidis, V. M. Axt, and T. Kuhn. Dynamics of exciton formation for near band gap excitations. Phys. Rev. B vol. 65, 035303 (2002).

23. S. Nikitine. J. Phys. Chem. Solids vol. 45, 949 (1984).

24. M. Kira and S. W. Koch. Exciton-Population Inversion and Terahertz Gain in Semiconductors Excited to Resonance. Phys. Rev. Lett. vol. 93, 076402 (2004).

25. L. V. Butov, L. S. Levitov, A. V. Mintsev, B. D. Simons, A. C. Gossard, and D. S. Chemla. Formation mechanism and low-temperature instability of exciton rings. Phys. Rev. Lett. vol. 92, 117404 (2004). 


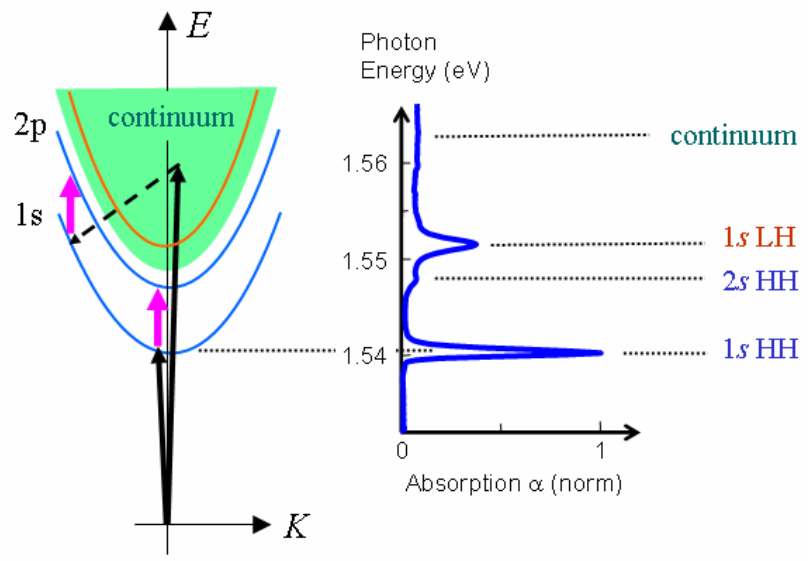

Fig. 1. Left: Illustration of the $e-h$ pair energy dispersion vs. the pair's center-of-mass momentum K. Black arrows: interband excitation, Magenta arrows: intra-excitonic transitions. Exciton formation is indicated by the dashed arrow. Right: measured near-infrared absorption spectrum of the quantum well sample studied here, along with assignments of the main peaks. 
Figure 2

R.A. Kaindl et al. - “Applications of Ultrafast Terahertz...”

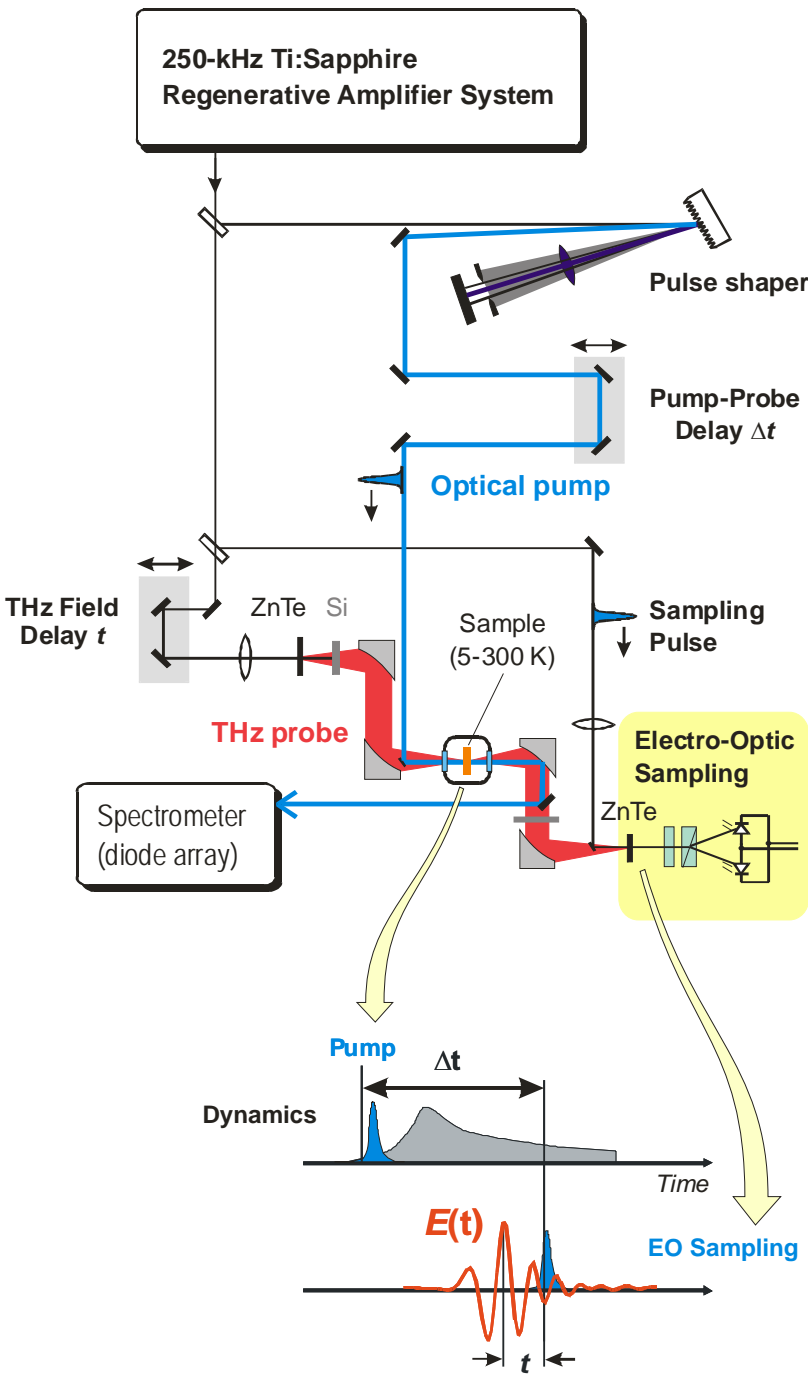

Fig. 2. Experimental setup for optical-pump THz-probe spectroscopy at $250 \mathrm{kHz}$ repetition rate. The $\mathrm{THz}$ probe pulse is used in transmission geometry, with the quantum well sample mounted in an optical cryostat equipped with mylar windows. The THz section of the setup is purged in dry nitrogen to avoid absorption from ambient air. 


\section{Figure 3}

R.A. Kaindl et al. - “Applications of Ultrafast Terahertz...”

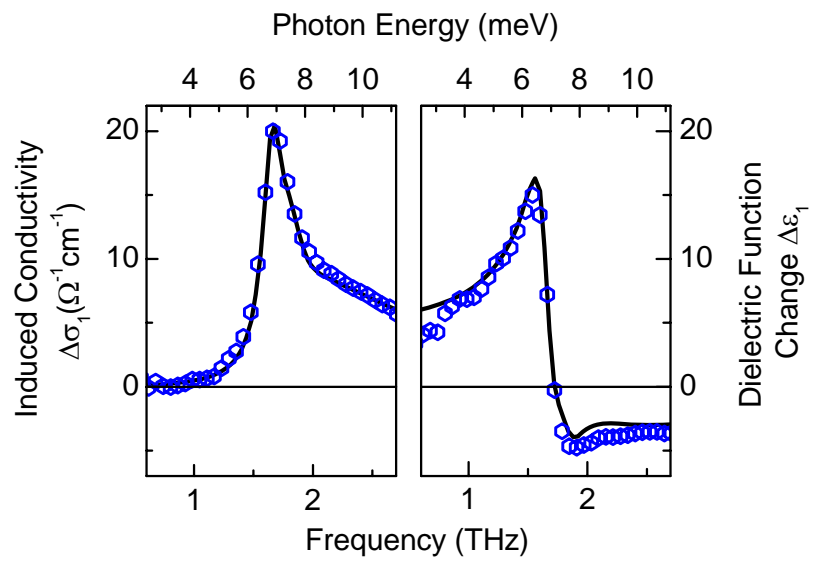

Fig. 3. Photo-induced $\mathrm{THz}$ response (open symbols) after resonant excitation of $1 s-\mathrm{HH}$ excitons in GaAs quantum wells. Left panel: induced real part of conductivity, $\Delta \sigma_{1}$. Right panel: change of the real part of the dielectric function, $\Delta \varepsilon_{1}$. The spectra were measured at lattice temperature $T=6 \mathrm{~K}$ and shortly after photoexcitation (delay time $\Delta t=10 \mathrm{ps}$ ) following the dephasing of the initially coherent interband excitonic polarization. Solid lines: model functions of the intra-excitonic response for an exciton density $n_{X}=2.7 \times 10^{10} \mathrm{~cm}^{-2}$. 

Photon Energy (meV)

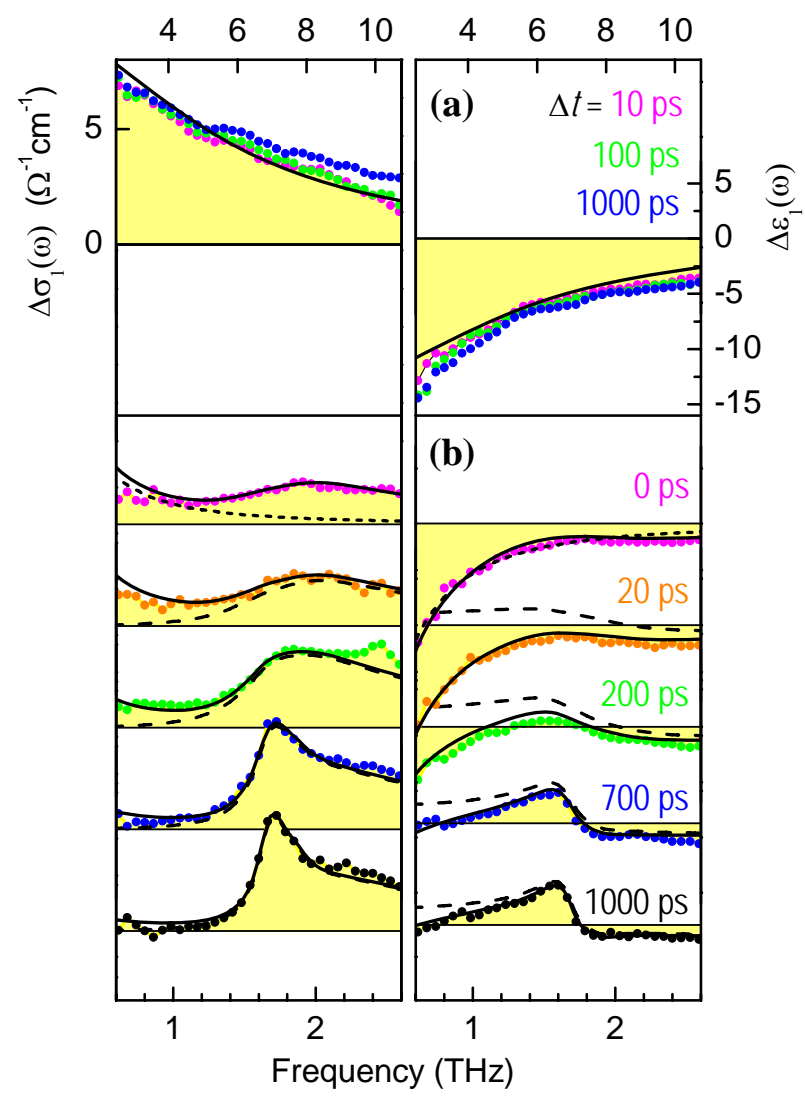

Fig. 4. Transient $\mathrm{THz}$ response (solid dots) after photoexcitation into the continuum of unbound states, for different pump-probe delays $\Delta t$ as indicated. All curves in each panel are scaled equally. (a) $T=300 \mathrm{~K}$ lattice temperature, photoexcitation at $1.555 \mathrm{eV}$ (87 meV above the exciton line). Black line: Drude model for $n_{\mathrm{eh}}=2.1 \times 10^{10} \mathrm{~cm}^{-2}$ and $\Gamma_{\mathrm{D}}=2 \pi \times 1.3 \mathrm{THz}$. (b) $T=6 \mathrm{~K}$ and photoexcitation at $1.561 \mathrm{eV}$, which is $21 \mathrm{meV}$ above the $1 \mathrm{~s} \mathrm{HH}$ exciton. Solid lines: two-component model as described in the text, corresponding to an initial excitation density of $1 \times 10^{10} \mathrm{~cm}^{-2}$. Dashed lines: exciton component. Dotted line: best-attempt Drude model fit for $\Delta t=0 \mathrm{ps}$, with $n_{\mathrm{eh}}=4.7 \times 10^{9} \mathrm{~cm}^{-2}$ and $\Gamma_{\mathrm{D}}=2 \pi \times 0.35 \mathrm{THz}$. 
Figure 5

R.A. Kaindl et al. - “Applications of Ultrafast Terahertz...”

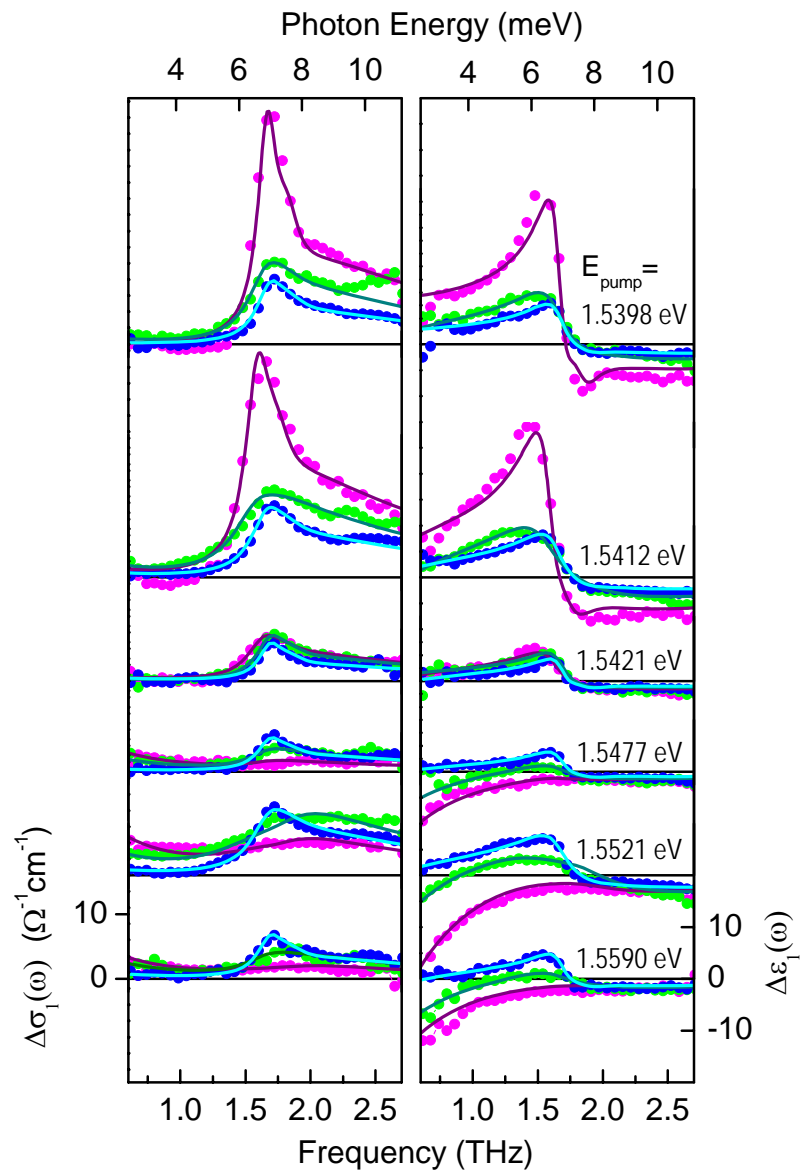

Fig. 5. Pump photon energy ( $\left.E_{\text {pump}}\right)$ dependence of the transient $\mathrm{THz}$ response, for three different pump-probe time delays each of $\Delta t=0$ ps (magenta), 200 ps (green), and 900 ps (blue). All curves in each panel are equally scaled, and shifted vertically as indicated by black horizontal lines. 
Figure 6 R.A. Kaindl et al. - “Applications of Ultrafast Terahertz...”

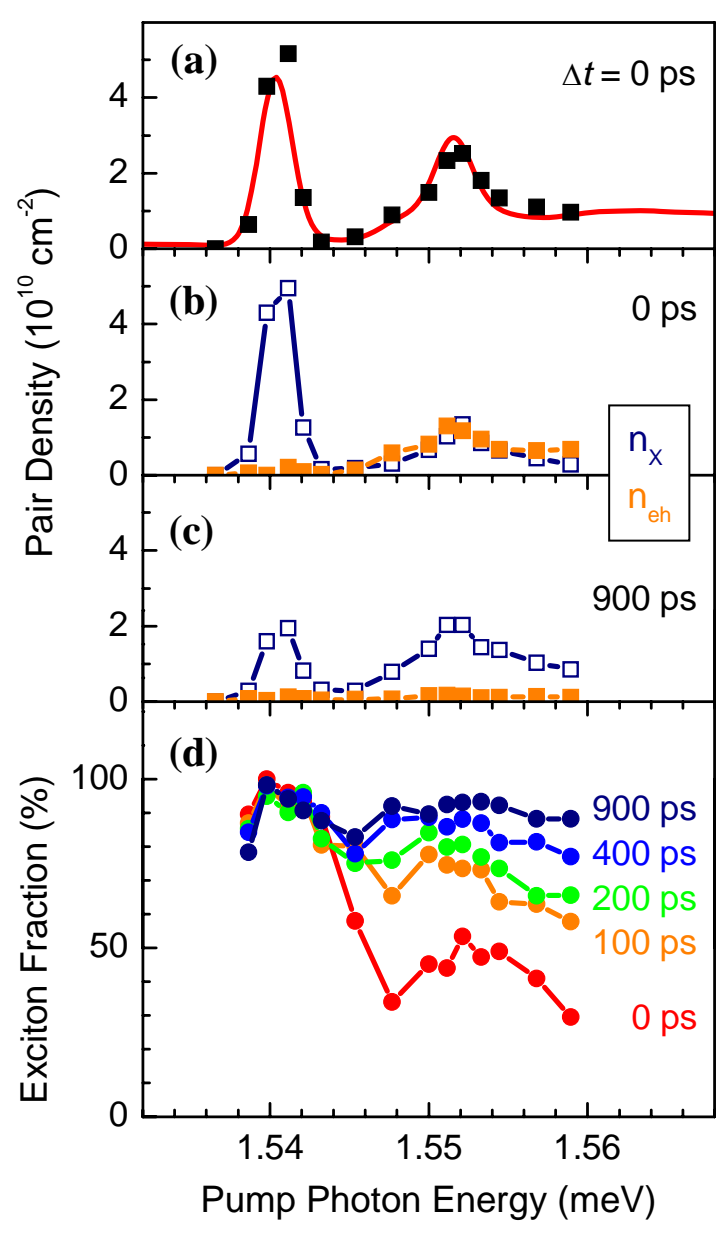

Fig. 6. Pump photon energy and time dependence of the pair densities, extracted via the twocomponent model from the data in Fig. 5. (a) Total pair density (squares) at $\Delta t=0$ ps. Red line: the near-IR absorption spectrum from Fig. 1 after convolution with a Gaussian of 2.2 meV width (FWHM). (b),(c) exciton density $n_{\mathrm{X}}$ and free carrier density $n_{\mathrm{eh}}$ for two different time delays. (d) exciton fraction $f$ for different time delays. 


\section{DISCLAIMER}

This document was prepared as an account of work sponsored by the United States Government. While this document is believed to contain correct information, neither the United States Government nor any agency thereof, nor The Regents of the University of California, nor any of their employees, makes any warranty, express or implied, or assumes any legal responsibility for the accuracy, completeness, or usefulness of any information, apparatus, product, or process disclosed, or represents that its use would not infringe privately owned rights. Reference herein to any specific commercial product, process, or service by its trade name, trademark, manufacturer, or otherwise, does not necessarily constitute or imply its endorsement, recommendation, or favoring by the United States Government or any agency thereof, or The Regents of the University of California. The views and opinions of authors expressed herein do not necessarily state or reflect those of the United States Government or any agency thereof, or The Regents of the University of California.

Ernest Orlando Lawrence Berkeley National Laboratory is an equal opportunity employer.

Ernest Orlando Lawrence Berkeley National Laboratory 